\title{
Terrestrial Rain Attenuation Time Series Synthesizers for Tropical Regions
}

\author{
F. J. A. Andrade, M. E. C. Rodrigues, L. A. R. da Silva Mello \\ Center for Telecommunication Studies - Catholic University of Rio de Janeiro Rio de Janeiro, Brazil, \\ smello@cetuc.puc-rio.br
}

\begin{abstract}
The aim of this paper is to present and validate rain attenuation time series synthesizers relying on the Maseng-Bakken principle for terrestrial links in tropical areas. Data obtained in experimental measurements at four links operating at $15 \mathrm{GHz}$ in São Paulo, Brazil, are used to parameterize the synthesizers. Two models are tested with respect to long-term statistics including cumulative distributions of rain attenuation, fade durations and fade slope.
\end{abstract}

Index Terms - propagation modeling, rain attenuation, time series generator, time series synthesizers.

\section{INTRODUCTION}

Rain attenuation is the main cause of unavailability in fixed terrestrial radio systems operating at frequency of above $10 \mathrm{GHz}$ in tropical and equatorial areas. Propagation impairments are expected to be quite severe and signal outage and performance standards requirements may be difficult to achieve in these regions. Due to these adverse propagation conditions, Fade Mitigating Techniques (FMT) [1] are often required.

To design and optimize FMT, the knowledge of the cumulative distribution of rain attenuation, commonly used in link design, is not enough. The characterization of the dynamic behavior of the propagation channel, as provided by fade durations and fade slope statistics, is also required. This need can be fulfilled by the introduction of time series of propagation impairments in system simulation.

An alternative to using real data collected from propagation experiments is to generate typical fading time-series, making use of climatological characteristics as well as geometrical and radiowave parameters of the link.

Two long-term rain attenuation time series synthesizers are presented and tested in this paper, both based on Maseng-Bakken (MB) theory [2]: the first one is the so-called Enhanced MB model (EMB) [3], [4], originally developed for satellite systems in temperate climates, whereas the other one is an modified version, adapted for terrestrial links, referred as the TMB model.

The aim of the study presented in this paper is to test, compare and validate these time series synthesizers for tropical regions, using data measured in four terrestrial radio links operating at 15 $\mathrm{GHz}[5]$. 


\section{CHANNEL MODELS DESCRIPTION}

\section{A. Maseng-Bakken and Enhanced Maseng-Bakken Models}

Maseng and Bakken have proposed a stochastic model of rain attenuation relying on the first order Markov theory in the eighties [2]. This concept has been applied to generate time-series of rain attenuation for satellite communication systems operating at Ku-band and above [6], [7]. The model has been improved and widely validated for temperate European climates by researchers from ONERA-CNES [3], [4], [8].

Maseng and Bakken have made two main hypotheses concerning the rain attenuation process $A_{\text {rain }}$ :

- The long-term distribution of rain attenuation is log-normal, characterized by two parameters: the mean $m$ and the standard deviation $\sigma$ of its natural logarithm;

- Rain attenuation can be transformed into a first order stationary Markov process using the nonlinear transformation:

$$
X=\left(\ln A_{\text {rain }}-m\right) / \sigma
$$

The dynamics of the rain attenuation process is described by a third parameter, $\beta$. The original model synthesizes just periods of rain. So, prior to the retrieval of parameters, the periods of time with attenuation values below a certain threshold must be excluded from the experimental time series. Fig. 1 presents the principle of the model.

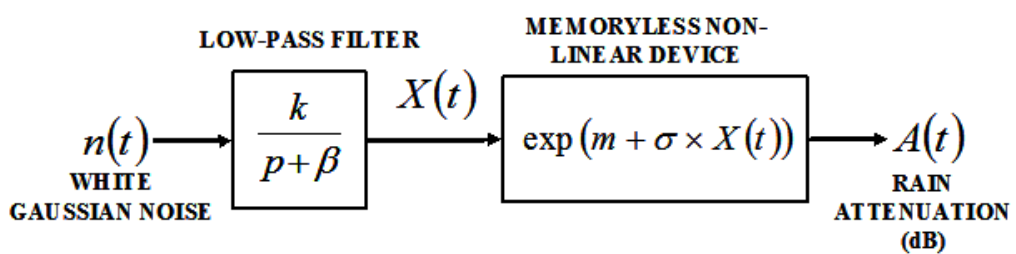

Fig. 1. Principle of the Maseng-Bakken model [3].

To be able to synthesize both periods of rain and of no rain, the EMB model was developed [3]. For this model, input parameters are retrieved from the attenuation time series (i.e., not conditioned to a threshold level) and the log-normal parameters $m$ and $\sigma$ can be derived from the long-term Complementary Cumulative Distribution Function (CCDF) by using a curve fitting method. If the experimental long-term CCDF is not available for a given link, the rain attenuation distribution may be predicted for the frequency of interest using the Rec. ITU-R P.618-7. A technique for $\beta$ assessment is discussed with detail in [9]. Besides the three parameters, the EMB model included a fourth one, an attenuation offset $A_{\text {off }}$ that is subtracted from the synthesized time series to improve the dynamics of the model [10]. Fig. 2 presents the principle of the EMB model.

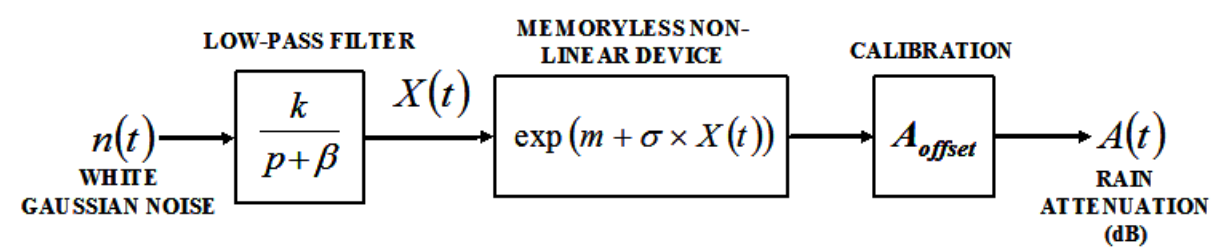

Fig. 2. Principle of the Enhanced Maseng-Bakken model [9]. 


\section{B. Terrestrial Enhanced Maseng-Baken model}

The TMB model, proposed in [11], is implemented by using the same parameters of EMB model. The difference is in the parameter extraction procedure. The log-normal parameters $m$ and $\sigma$ are derived from the long-term Complementary Cumulative Distribution Function (CCDF) of rain attenuation by using a curve fitting that minimize the RMS error in $\mathrm{dB}$ between experimental and theoretic synthesized attenuation CCDFs. $A_{\text {off }}$ is the attenuation value corresponding to $p \%$ of time, obtained from the CCDF having $m$ and $\sigma$ parameters. The $p$ value was set to $10 \%$, the upper limit of the range of time percentages used in the curve fitting. This value was derived from the experimental rain attenuation CCDFs of the links.

\section{APPLICATION WITH PROPAGATION DATA COLLECTED IN BRAZIL}

\section{A. Brazilian Terrestrial Data}

Time series of rain attenuation were continuously recorded from four terrestrial links located in São Paulo, Brazil. The operating frequencies, path lengths and experiment durations are given in Table I. The experimental setup included a tipping bucket raingauge with $0.1 \mathrm{~mm}$ capacity and a data acquisition unit that samples the AGC voltage of each receiver each 1 or 10 seconds, according to the link, storing the data together with the date and time of each raingauge tip. The data files were then processed to convert AGC voltage into received power levels [5].

TABLE I. LINK PARAMETERS

\begin{tabular}{ccccc}
\hline Link & Path Length $(\mathbf{k m})$ & Frequency $(\mathbf{G H z})$ & Measurement Period & Sampling Frequency $(\mathbf{H z})$ \\
\hline Bradesco (BD) & 12.8 & 15 & 2 years & 0.1 \\
Cenesp15 (CN15) & 12.8 & 15 & 2 years & 0.1 \\
Scania (SC) & 18.4 & 15 & 1 year & 0.1 \\
Paranapiacaba (PR) & 42.9 & 15 & 2 years & 1.0 \\
\hline
\end{tabular}

\section{B. Retrieval of Channel Models Parameters}

Table II presents parameters for the EMB and TMB models for these data. The log-normal adjust was made for the range of time percentages from 0.01 to $10 \%$ for Bradesco and Cenesp15 links, from 0.03 to $10 \%$ for the Scania link and from 0.1 to $10 \%$ for Paranapiacaba link.

TABLE II. MODEL PARAMETERS

\begin{tabular}{cccccc}
\hline Link & Model & $\boldsymbol{m}$ & $\boldsymbol{\sigma}$ & $\boldsymbol{A}_{\text {off }}(\mathbf{d B})$ & $\boldsymbol{\beta}\left(\mathbf{s}^{-\mathbf{1}}\right)$ \\
\hline \multirow{2}{*}{ Bradesco (BD) } & EMB & -3.74 & 2.07 & 0.00 & $1.16 \mathrm{e}-4$ \\
& TMB & -0.96 & 1.24 & 1.87 & $1.26 \mathrm{e}-4$ \\
Cenesp15 (CN15) & EMB & -2.91 & 1.88 & 0.60 & $1.20 \mathrm{e}-4$ \\
& TMB & -0.26 & 1.09 & 3.13 & $1.52 \mathrm{e}-4$ \\
Scania (SC) & EMB & -3.47 & 2.13 & 0.40 & $6.43 \mathrm{e}-5$ \\
& TMB & -1.60 & 1.54 & 1.46 & $6.59 \mathrm{e}-5$ \\
Paranapiacaba (PR) & EMB & -3.02 & 2.23 & 0.80 & $5.60 \mathrm{e}-5$ \\
& TMB & -0.47 & 1.35 & 3.54 & $6.78 \mathrm{e}-5$ \\
\hline
\end{tabular}




\section{RESULTS}

Rain attenuation time series were synthesized by EMB and TMB models for each link with the same sampling frequency of the experimental data. In order to verifying models stability, five time series were generated for the links. The time series length of BD, CN15 and SC links is 50 years while for PR link the length is 5 years. To compare synthesized and experimental data and compute the errors it was considered the first synthesized time series by each model for each link instead of considering the best time series among the five, what as was presented in [11].

\section{A. CCDF of Rain Attenuation}

Figure 3 to Figure 6 present the attenuation CCDFs of the time series synthesized by the two models and the experimental distribution for the four links.

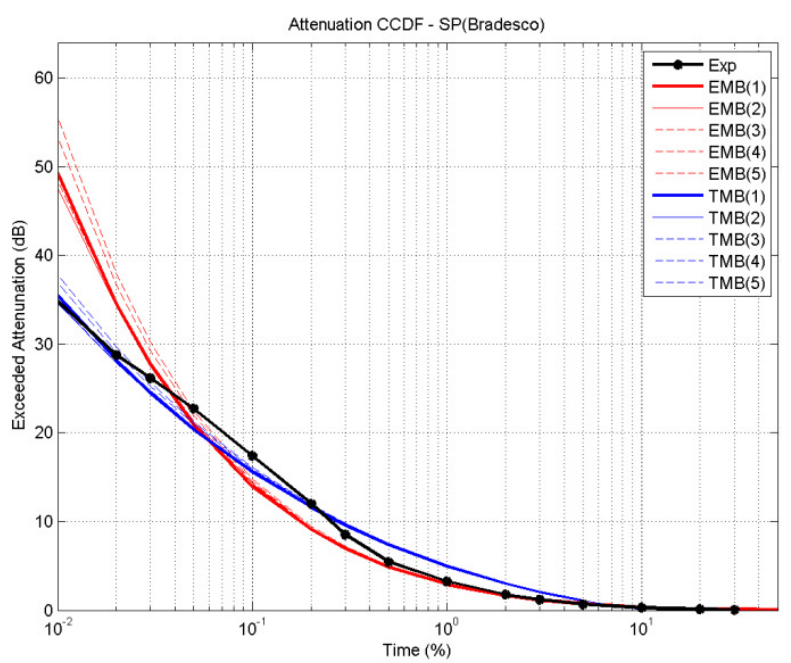

Fig. 3. Comparison between CCDF of rain attenuation of synthesized and experimental data from Bradesco link.

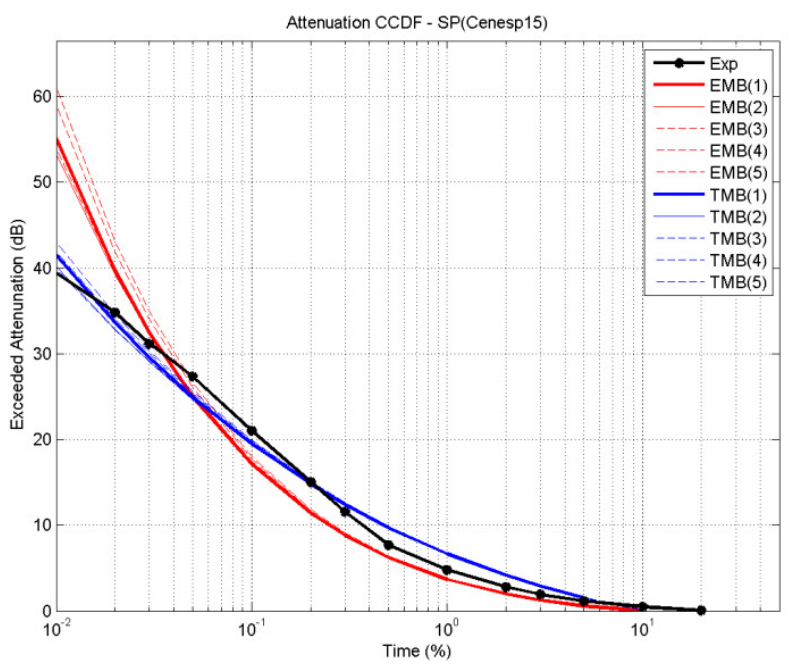

Fig. 4. Comparison between CCDF of rain attenuation of synthesized and experimental data from Cenesp15 link. 


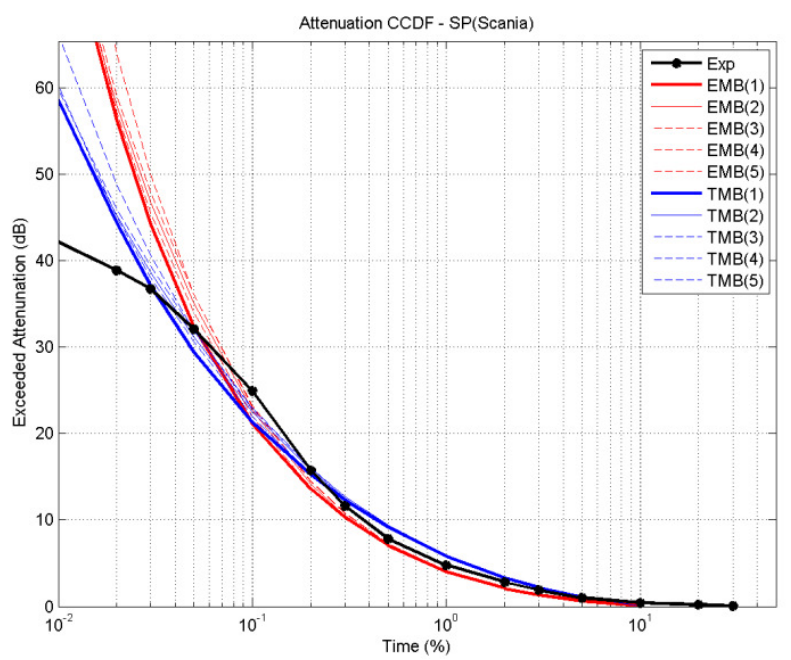

Fig. 5. Comparison between CCDF of rain attenuation of synthesized and experimental data from Scania link.

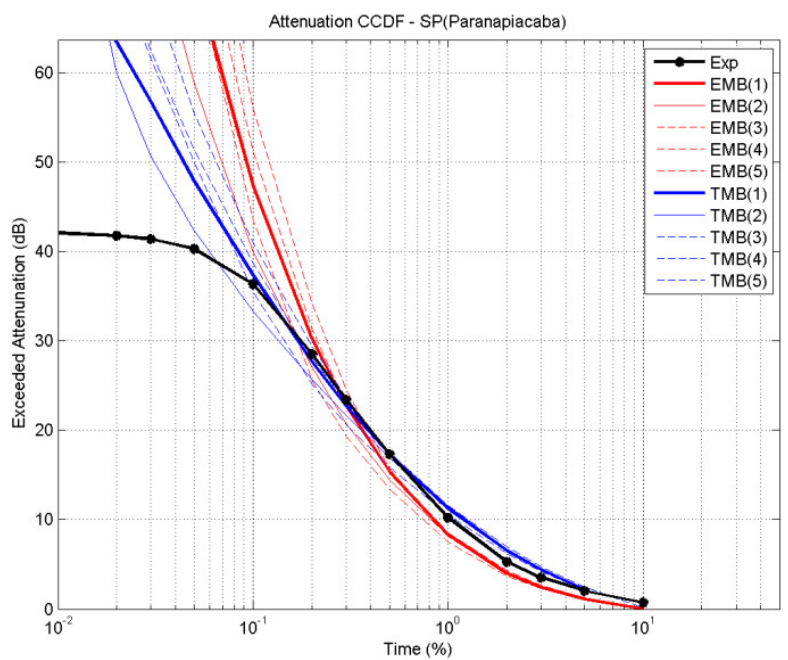

Fig. 6. Comparison between CCDF of rain attenuation of synthesized and experimental data from Paranapiacaba link.

It can be observed that for low values of attenuation the EMB CCDF is closer to experimental data for Bradesco and Cenesp15 links. However, for the high values of attenuation associated with low percentages of time, that are relevant for the design of fade mitigation systems for tropical areas, the TMB CCDF is closer to experimental data than EMB CCDF for the four links.

Paranapiacaba is the longest link and its parameters had to be extracted from a narrower range of time percentages because its link margin is about $40 \mathrm{~dB}$.

Figure 7 shows the mean absolute errors of each model for the four links as a function of time percentage. It can be observed that TMB model provides lower values of error than EMB model for time percentages below 0.3 . 


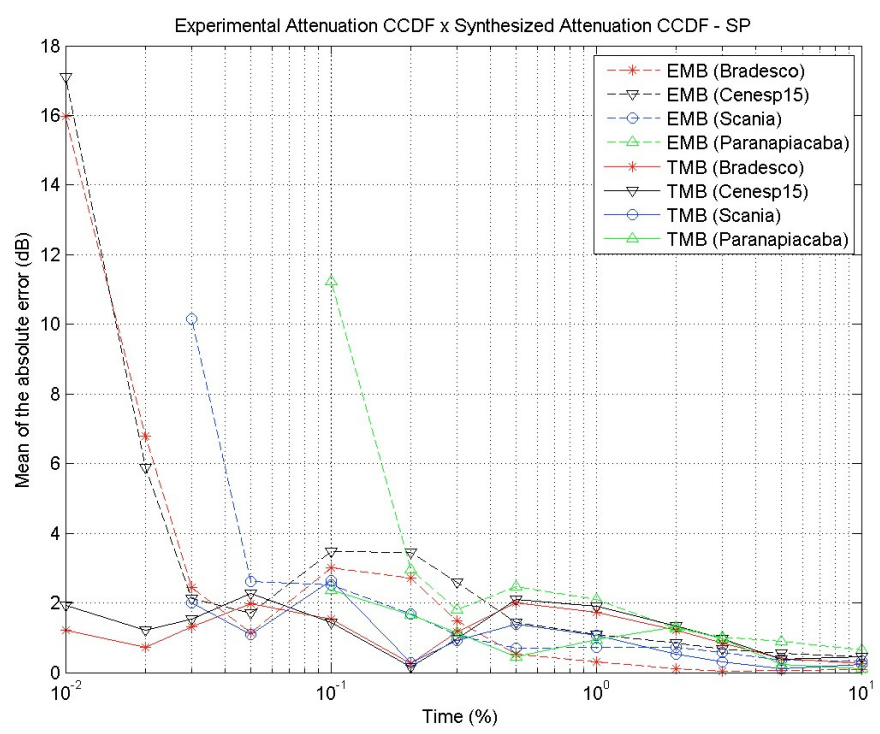

Fig. 7. Comparison between mean values of absolute errors of synthesized data.

Table III shows the RMS errors obtained from the comparison between experimental CCDF and CCDF of the synthesized data of the first synthesized time series of each link.

TABLE III. RMS ERROR FOR ATTENUATION CCDF

\begin{tabular}{|c|c|c|c|c|}
\hline Link & Model & & Range & \\
\hline \multirow{3}{*}{ Bradesco (BD) } & & $0.01-5 \%$ & $0.01-1 \%$ & $0.01-0.2 \%$ \\
\hline & EMB & 0.17 & 0.19 & 0.22 \\
\hline & TMB & 0.21 & 0.16 & 0.07 \\
\hline \multirow{3}{*}{ Cenesp15 (CN15) } & & $0.01-5 \%$ & $0.01-1 \%$ & $0.01-0.2 \%$ \\
\hline & EMB & 0.26 & 0.22 & 0.21 \\
\hline & TMB & 0.17 & 0.13 & 0.06 \\
\hline \multirow{3}{*}{ Scania (SC) } & & $0.03-5 \%$ & $0.03-1 \%$ & $0.03-0.2 \%$ \\
\hline & EMB & 0.18 & 0.14 & 0.14 \\
\hline & TMB & 0.11 & 0.11 & 0.09 \\
\hline \multirow{3}{*}{ Paranapiacaba (PR) } & & $0.1-5 \%$ & $0.1-1 \%$ & $0.1-0.2 \%$ \\
\hline & EMB & 0.25 & 0.16 & 0.19 \\
\hline & TMB & 0.10 & 0.05 & 0.03 \\
\hline
\end{tabular}

The errors were computed over 3 different ranges of time percentages using the RMS value of test variable values given in Rec. ITU-R P.311-12 in each range. For a given method and for each percentage of time, the value of the test variable $V_{i}$ is given by

$$
V_{i}=\left\{\begin{array}{cc}
\left(A_{m} / 10\right)^{0.2} \ln \left(A_{s} / A_{m}\right) & \text { for } A_{m}<10 \mathrm{~dB} \\
\ln \left(A_{s} / A_{m}\right) & \text { for } A_{m} \geq 10 \mathrm{~dB}
\end{array}\right.
$$

where $A_{m}(\mathrm{~dB})$ is the measured attenuation and $A_{s}(\mathrm{~dB})$ is the synthesized attenuation.

It is possible to observe that TMB model always provides better results than the EMB model especially for the ranges associated to high values of attenuation.

We can note that the values of error for EMB model applied to the 4 terrestrial links operating at 15 $\mathrm{GHz}$ in a tropical region are very close to values obtained for beacon data of 6 links operating at frequencies from 19 to $50 \mathrm{GHz}$ in temperate areas [12]. 


\section{B. Number and Time of Events}

Figure 8 to Figure 11 present the fade duration distributions for 10 and $25 \mathrm{~dB}$ attenuation thresholds of experimental data and data synthesized by each model for the four links.
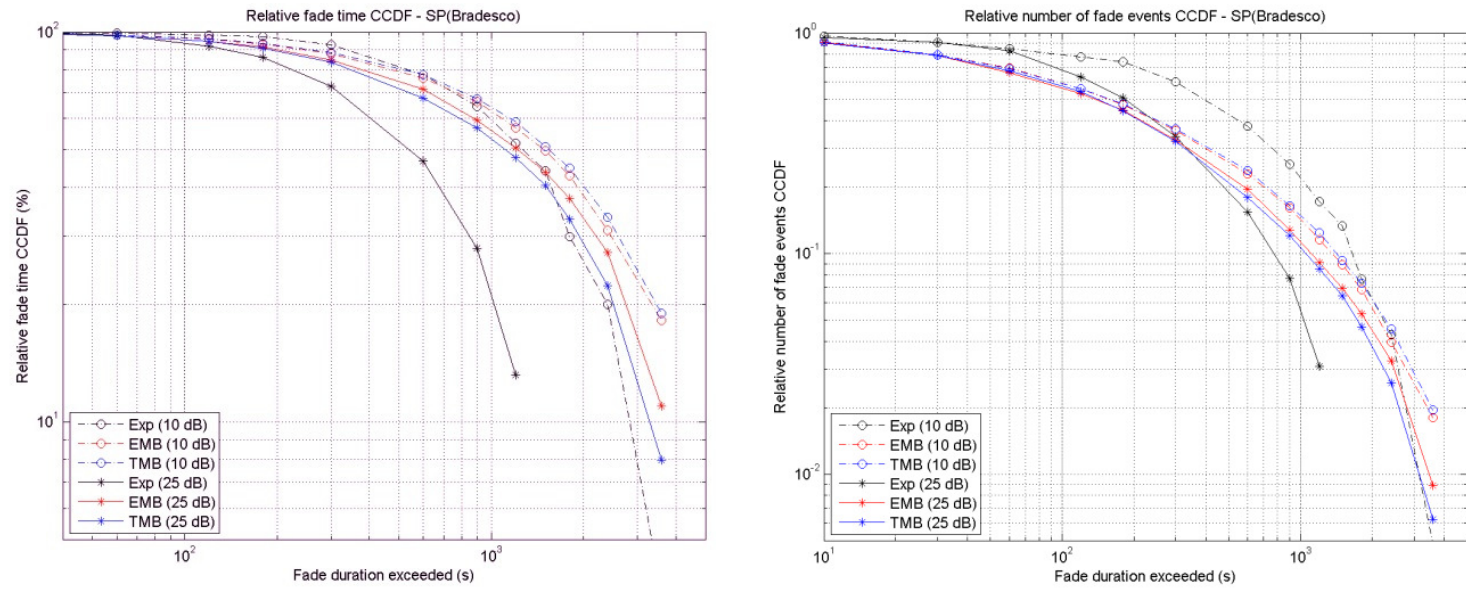

Fig. 8. Fade duration CCDFs from Bradesco link.
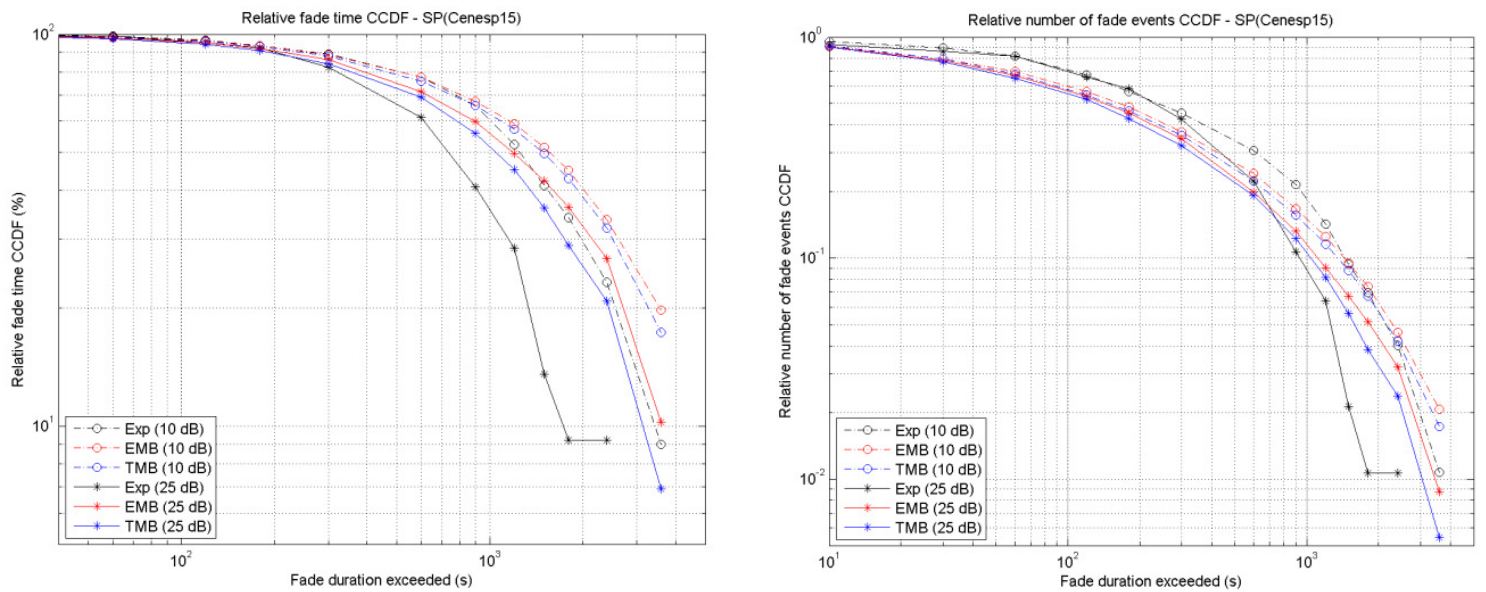

Fig. 9. Fade duration CCDFs from Cenesp15 link.
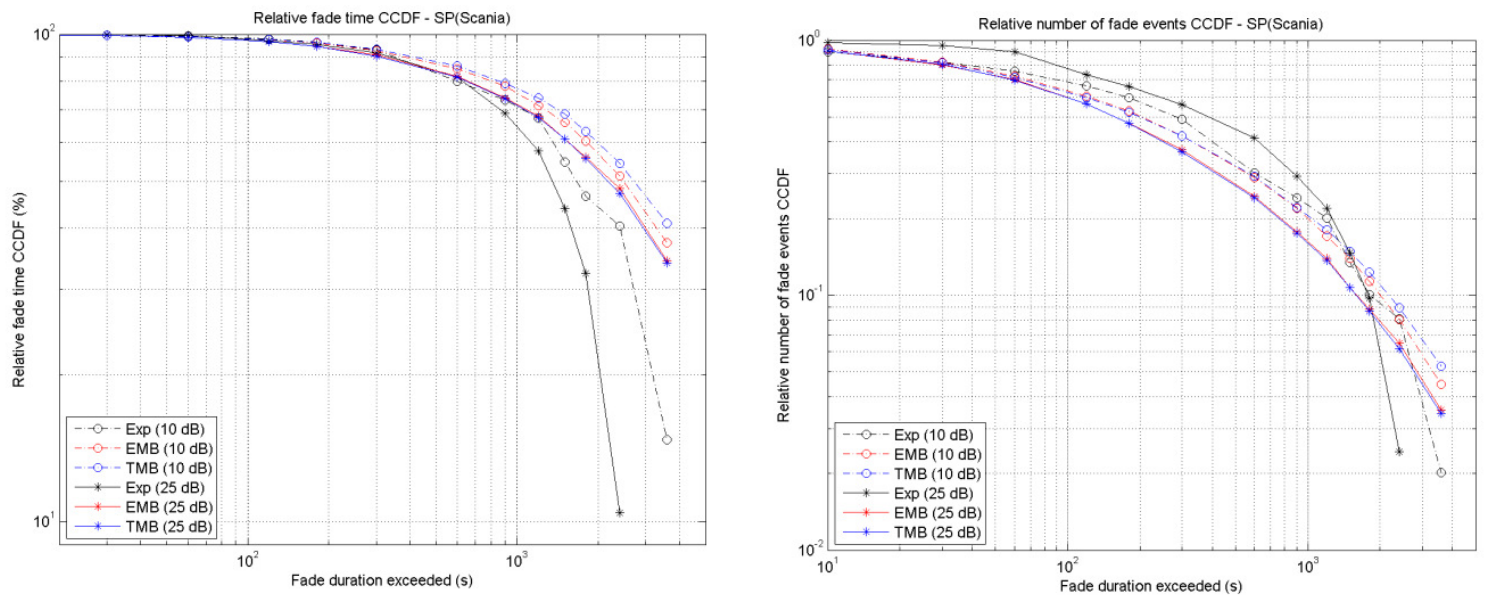

Fig. 10. Fade duration CCDFs from Scania link. 

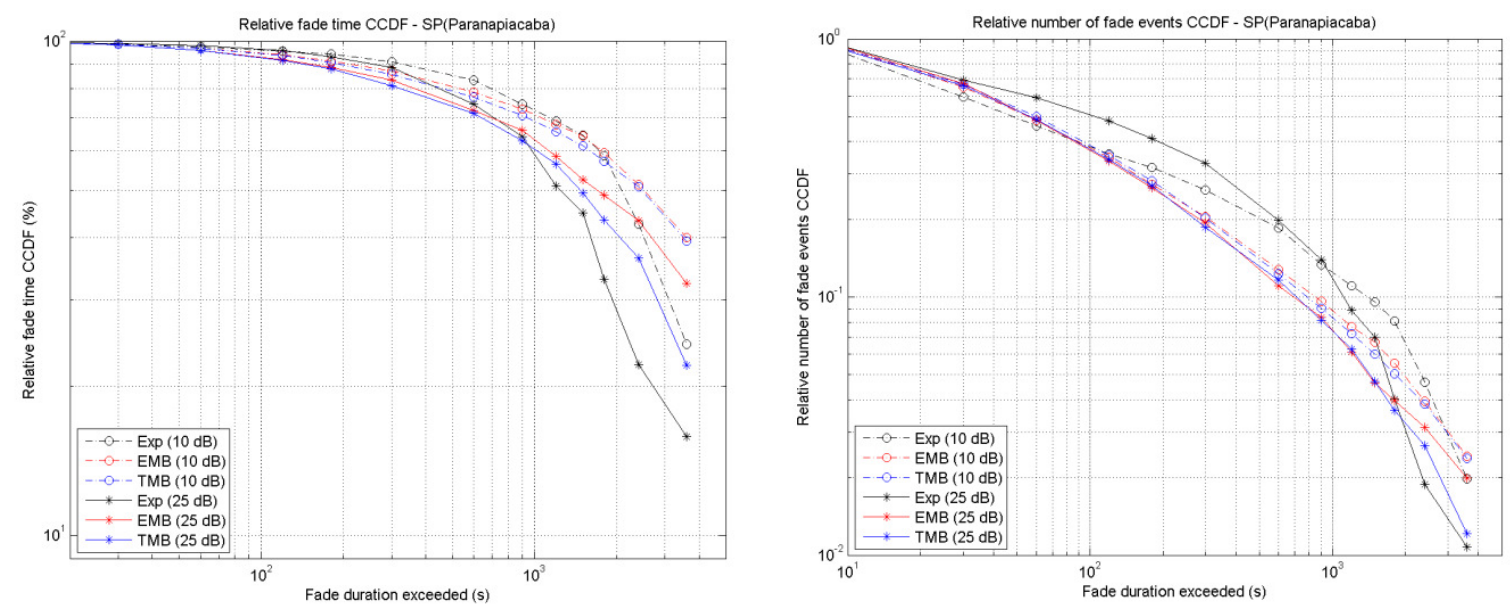

Fig. 11. Fade duration CCDFs from Paranapiacaba link.

Tables IV and V show the RMS errors of test variables obtained from the comparison between the number and time of fades CCDFs of experimental and synthesized data. To be consistent with Rec. ITU-R P.311-12, the error for relative fade time is calculated using the test variable $\varepsilon_{T}$ for the total fraction of fade time $F(d>D \mid a>A)$ due to fades of duration $d$ longer than $D$ (s), given that the attenuation $a$ is greater than $A(\mathrm{~dB})$. The test variable is defined for each attenuation threshold $A$ and for each fade duration value $D$ defined by ITU and is calculated as

$$
\varepsilon_{T}(D, A)=\ln \left(\frac{100-F_{s}(D \mid A)}{100-F_{m}(D \mid A)}\right)
$$

For the error for relative number of fades, the test variable $\varepsilon_{N}$ is calculated according the probability $P(d>D \mid a>A)$ of occurrence of fades of duration $d$ longer than $D(\mathrm{~s})$, given that the attenuation $a$ is greater than $A(\mathrm{~dB})$.

$$
\varepsilon_{N}(D, A)=\ln \left(\frac{P_{s}(D \mid A)}{P_{m}(D \mid A)}\right)
$$

The RMS values were calculated for each attenuation threshold, for all thresholds and for thresholds greater or equal to $10 \mathrm{~dB}$.

TABLE IV. RMS ERROR FOR RELATIVE FADE TIME

\begin{tabular}{ccccccccc}
\hline $\begin{array}{c}\text { Thresholds } \\
\text { (dB) }\end{array}$ & \multicolumn{2}{c}{ Bradesco } & \multicolumn{2}{c}{ Cenesp15 } & \multicolumn{2}{c}{ Scania } & \multicolumn{2}{c}{ Paranapiacaba } \\
EMB & TMB & EMB & TMB & EMB & TMB & EMB & TMB \\
\hline 3 & 0.51 & 0.45 & 0.38 & 0.38 & 0.16 & 0.10 & 0.20 & 0.20 \\
5 & 0.34 & 0.31 & 0.16 & 0.16 & 0.20 & 0.22 & 0.14 & 0.17 \\
10 & 0.53 & 0.51 & 0.19 & 0.24 & 0.22 & 0.27 & 0.22 & 0.26 \\
15 & 0.45 & 0.43 & 0.23 & 0.24 & 0.17 & 0.18 & 0.33 & 0.37 \\
20 & 0.37 & 0.35 & 0.30 & 0.28 & 0.26 & 0.28 & 0.26 & 0.29 \\
25 & 0.49 & 0.42 & 0.35 & 0.35 & 0.40 & 0.41 & 0.36 & 0.37 \\
30 & 0.60 & 0.60 & 0.36 & 0.23 & 0.39 & 0.39 & 0.37 & 0.39 \\
35 & - & - & 0.40 & 0.33 & 0.61 & 0.60 & 0.40 & 0.44 \\
Total & 0.55 & 0.50 & 0.37 & 0.32 & 0.38 & 0.37 & 0.30 & 0.33 \\
Total ( $\geq 10 \mathrm{~dB})$ & 0.54 & 0.51 & 0.34 & 0.29 & 0.41 & 0.41 & 0.33 & 0.36 \\
\hline
\end{tabular}


TABLE V. RMS ERROR FOR RELATIVE NUMBER OF FADES

\begin{tabular}{ccccccccc}
\hline $\begin{array}{c}\text { Thresholds } \\
\text { (dB) }\end{array}$ & \multicolumn{2}{c}{ Bradesco } & \multicolumn{2}{c}{ Cenesp15 } & \multicolumn{2}{c}{ Scania } & \multicolumn{2}{c}{ Paranapiacaba } \\
\hline 3 & EMB & TMB & EMB & TMB & EMB & TMB & EMB & TMB \\
5 & 0.41 & 0.35 & 0.31 & 0.31 & 0.15 & 0.12 & 0.38 & 0.37 \\
10 & 0.28 & 0.26 & 0.13 & 0.12 & 0.10 & 0.11 & 0.15 & 0.16 \\
15 & 0.54 & 0.54 & 0.25 & 0.24 & 0.26 & 0.31 & 0.27 & 0.32 \\
20 & 0.63 & 0.61 & 0.27 & 0.21 & 0.34 & 0.32 & 0.42 & 0.47 \\
25 & 0.52 & 0.48 & 0.37 & 0.23 & 0.41 & 0.42 & 0.44 & 0.45 \\
30 & 0.48 & 0.44 & 0.73 & 0.60 & 0.47 & 0.47 & 0.45 & 0.39 \\
35 & 0.59 & 0.54 & 0.71 & 0.52 & 0.33 & 0.34 & 0.39 & 0.44 \\
Total & - & - & 0.49 & 0.42 & 0.27 & 0.27 & 0.43 & 0.52 \\
Total $(\geq 10 \mathrm{~dB})$ & 0.53 & 0.49 & 0.49 & 0.39 & 0.33 & 0.34 & 0.44 & 0.48 \\
\hline
\end{tabular}

The TMB model provides better results for fade duration statistics for two of the radio links considered. Most of the RMS values for relative fade time are similar to the values obtained for beacon in temperate areas [12].

\section{Fade Slope}

Figure 12 to Figure 15 present the fade slope distributions for two attenuation thresholds for each link. The fade slopes were obtained with a $30 \mathrm{~s}$ time interval.

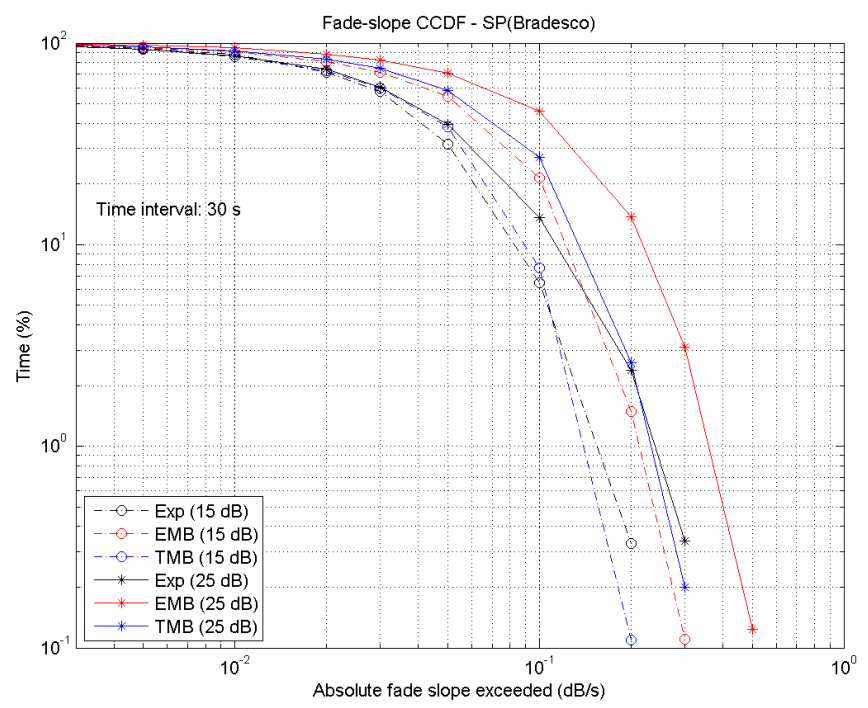

Fig. 12. Fade slope CCDF from Bradesco link. 


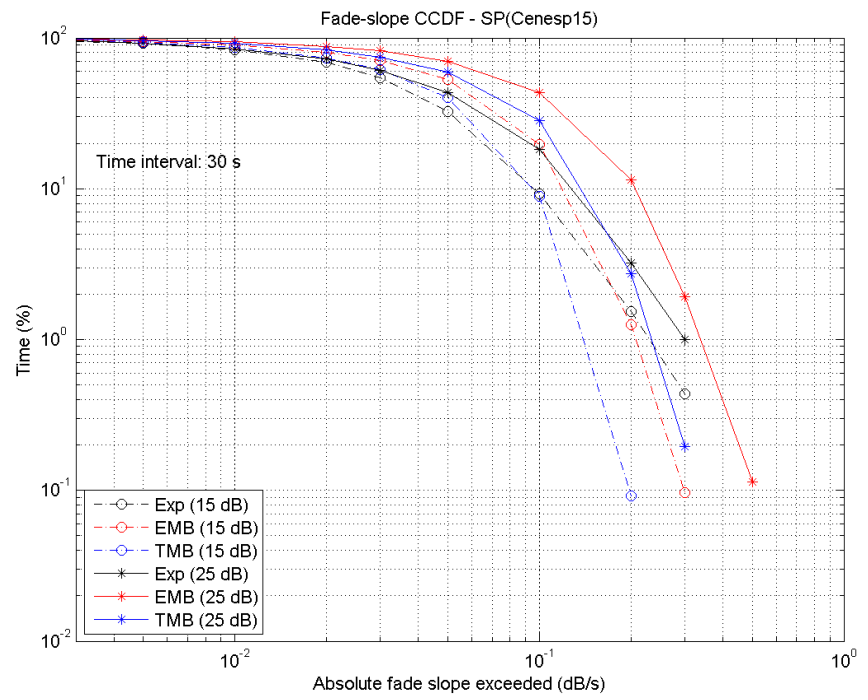

Fig. 13. Fade slope CCDF from Cenesp15 link.

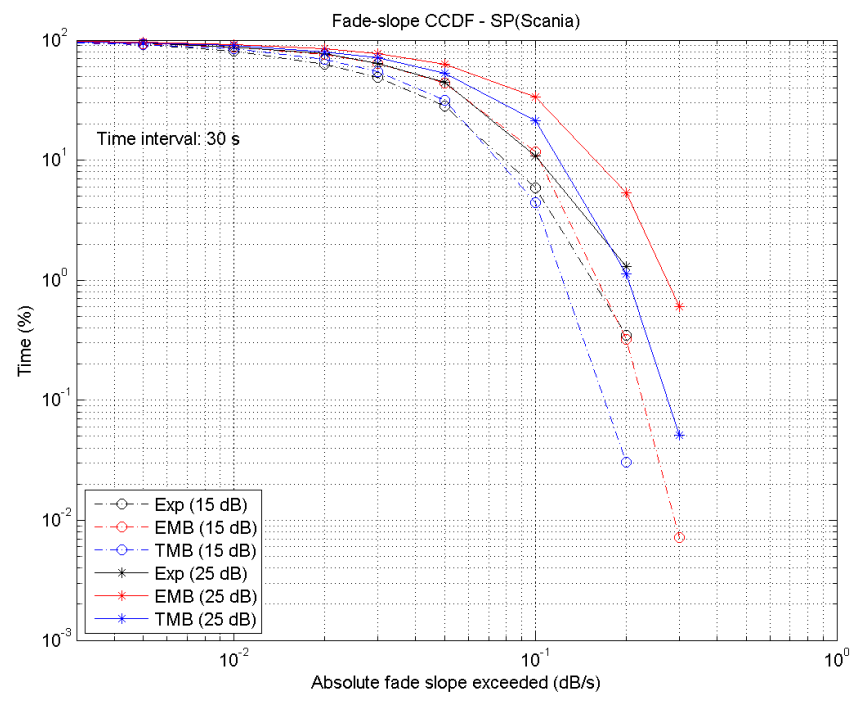

Fig. 14. Fade slope CCDF from Scania link.

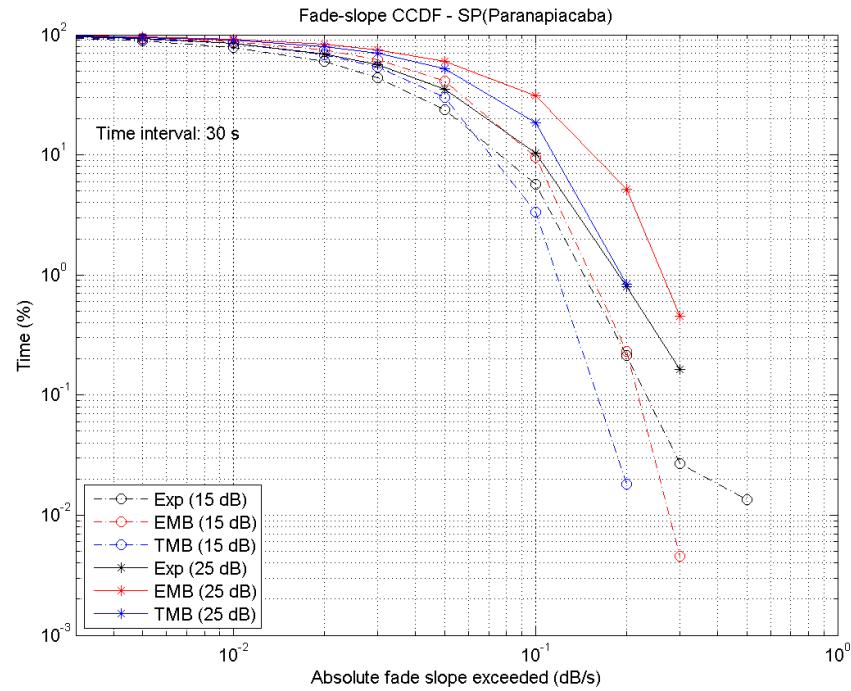

Fig. 15. Fade slope CCDF from Paranapiacaba link. 
Both models generally overestimate fade slope. However, it can be noted that for each threshold, TMB fade slope CCDFs are in general closer to experimental fade slope CCDF than EMB CCDFs.

Table VI shows the RMS errors of test variables obtained from the comparison between fade slope statistics of experimental and synthesized data according Rec. ITU-R P.311-12. For each attenuation threshold $A$ and for each fade slope value $\zeta$ defined by ITU, the test variable $\varepsilon_{F S}$ is calculated from the synthesized exceedence probability $P_{s}(\zeta \mid A)$ and the measured exceedence probability $P_{m}(\zeta \mid A)$ for each radio link as

$$
\varepsilon_{F S}(\zeta, A)=2 \cdot \frac{P_{s}(\zeta \mid A)-P_{m}(\zeta \mid A)}{P_{s}(\zeta \mid A)+P_{m}(\zeta \mid A)}
$$

Again, the RMS values were calculated for each attenuation threshold, for all thresholds and for thresholds greater or equal to $10 \mathrm{~dB}$.

TABLE VI. RMS ERROR FOR FADE SLOPES

\begin{tabular}{ccccccccc}
\hline $\begin{array}{c}\text { Thresholds } \\
\text { (dB) }\end{array}$ & \multicolumn{2}{c}{ Bradesco } & \multicolumn{2}{c}{ Cenesp15 } & \multicolumn{2}{c}{ Scania } & \multicolumn{2}{c}{ Paranapiacaba } \\
EMB & TMB & EMB & TMB & EMB & TMB & EMB & TMB \\
\hline 3 & 0.72 & 0.78 & 1.10 & 1.11 & 0.89 & 0.95 & 1.18 & 1.16 \\
5 & 0.76 & 0.89 & 1.01 & 1.05 & 0.65 & 0.77 & 1.07 & 1.08 \\
10 & 0.60 & 0.90 & 0.75 & 0.91 & 0.56 & 0.78 & 0.87 & 0.95 \\
15 & 0.80 & 0.33 & 0.48 & 0.81 & 0.66 & 0.54 & 0.75 & 0.97 \\
20 & 0.84 & 0.59 & 0.69 & 0.67 & 0.82 & 0.19 & 0.72 & 0.94 \\
25 & 0.92 & 0.29 & 0.75 & 0.45 & 0.78 & 0.64 & 0.63 & 0.64 \\
30 & 0.90 & 0.32 & 0.92 & 0.40 & 0.98 & 0.71 & 0.86 & 0.49 \\
35 & - & - & 1.01 & 0.95 & 0.96 & 0.68 & 1.13 & 0.95 \\
Total & 0.86 & 0.66 & 0.95 & 0.90 & 0.88 & 0.76 & 1.04 & 1.00 \\
Total ( $\geq 10 \mathrm{~dB})$ & 0.85 & 0.57 & 0.85 & 0.81 & 0.85 & 0.68 & 0.92 & 0.93 \\
\hline
\end{tabular}

The TMB model provides better results than the EMB model in Bradesco, Cenesp15 and Scania links especially when thresholds greater than or equal to $10 \mathrm{~dB}$ are considered. In this case, the values of RMS error for TMB model in tropical terrestrial links are in the range from 0.57 to 0.93 , similar to that observed for EMB model in temperate climates $(0.50$ to 0.92$)$ [12]. The agreement between the fade slope distributions derived from measured and synthesized data is slightly worse than the one that was obtained for fade duration statistics.

Paranapiacaba link, whose experimental data have sampling frequency of $1 \mathrm{~Hz}$, present similar results for both models. Although data from this link have a higher resolution, both models present higher values of error in this link. This indicates that the higher resolution of the experimental data does not guarantee a better performing of the models.

\section{CONCLUSIONS}

Parameters of two models for rain attenuation time series synthesizers were obtained for a group of four terrestrial links in São Paulo, Brazil. The models are modified versions of the Maseng-Bakken model developed in the eighties for earth space links. 
The statistics of attenuation level, fade duration and fade slope were derived from synthesized time series and tested against the corresponding ones derived from the experimental data. RMS errors are presented.

It is possible to conclude that both models may be applied for terrestrial links in tropical areas but TMB model, proposed in this paper, provides better results, particularly for deep fades, and may help in the design and optimization of FMT. This is important for tropical areas where high rainfall rates and, consequently, high attenuations due to rain are observed.

Results are particularly good for the cumulative distributions of attenuation. The results for the dynamic characteristics of rain attenuations, particularly fade slope are improved by the use of TMB model. This is an essential statistic for the design of FMT and will be further investigated. It was observed that, in some cases, the CCDF of attenuation are bimodal, so that a single lognormal may not be the most appropriate distribution to fit the experimental data in a tropical area. Other distributions are been considered. The bi-lognormal and Gamma distributions are being tested in an effort to improve the results.

\section{ACKNOWLEDGMENT}

This work was supported by CNPq under covenant 573939/2008-0 (INCT-CSF). The authors are indebted with Laurent Castanet and Guillaume Carrié from Onera, France, for the fruitful discussions concerning the EMB model and the ongoing collaboration between CETUC and Onera.

\section{REFERENCES}

[1] Arbesser-Rastburg et al., "Radiowave propagation modelling for new satcom services at Ku-band and above," COST 255 Final Report, ESA Publications Division, SP-1252, March 2002.

[2] T. Maseng, and P. M. Bakken, "A Stochastic Dynamic Model of Rain Attenuation," IEEE Trans. Com., Vol. 29, n’5, pp. 660-669, May 1981.

[3] F. Lacoste, M. Bousquet, L. Castanet, F. Cornet, and J. Lemorton, "Improvement of the ONERA-CNES rain attenuation time series synthesizer and validation of the dynamic characteristics of the generated fade events," Space Comm. Journal, Vol. 20, n1-2, 2005.

[4] J. Lemorton et al., "Development and validation of time series synthesizers of rain attenuation for Ka-band and Q/Vband satellite communication systems," Int. Journal of Satellite Comm. and Networking, V. 25, October 2007, pp 575601.

[5] L. A. R. Silva Mello, E. Costa, and R. S. L. Souza. Rain attenuation measurements at 15 and 18 GHz. Electronics Letters, London, v. 38, n. 4, p. 197-198, February 2002.

[6] B. C. Grémont and M. Filip, "Spatio-Temporal Rain Attenuation Model for Application To Fade Mitigation Techniques,” IEEE Trans. on Antennas and Propagation, Vol. 52, N5, pp. 1245-1256, May 2004.

[7] S.A. Kanellopoulos, A.D. Panagopoulos, and J.D. Kanellopoulos, "Calculation of the dynamic input parameter for a stochastic model simulating rain attenuation: a novel mathematical approach," IEEE Trans. on Antennas and Propagation, Vol.5, n¹1, November 2007, pp.3257-3264.

[8] F. Lacoste, M. Bousquet, F. Cornet, L. Castanet, and J. Lemorton, "Classical and On-Demand Rain Attenuation Time Series Synthesis: Principle and Applications," Proceedings of 24th AIAA-ICSSC Conference, San Diego, California, June 2006.

[9] G. Carrié, F. Lacoste, and L. Castanet, "A Robust Estimator of the Dynamic Characteristics of Rain Attenuation Events," Proceedings of 2nd JA2310 SatNEx Workshop, Oberpfaffenhofen, Germany, April 2008.

[10] M. E. C. Rodrigues and L. A. R. da Silva Mello, "Slant Path Rain Attenuation Synthesizers for Tropical and Equatorial Regions," presented at 14th International Symposium on Antenna Technology and Applied Electromagnetics, Ottawa, Canada, July 5-8, 2010.

[11] F. J. A. Andrade, M. E. C. Rodrigues, and L. A. R. da Silva Mello, "Terrestrial Rain Attenuation Time Series Synthesizers for Tropical Regions," presented at MOMAG 2010, Vila Velha, Brazil, August 29-September 1, 2010.

[12] G. Carrié, F. Lacoste, and L. Castanet, "Validation of Rain Attenuation Time Series Synthesizers for Temperate Area On the Enhanced Maseng-Bakken Model," in Proc. IWSSC' 08, Toulouse, France, October 2008. 